\title{
ÉLEVAGE, BIODIVERSITÉ ET ÉMERGENCES DE PANDÉMIES
}

\section{LIVESTOCK, BIODIVERSITY AND EMERGENCE OF PANDEMICS}

Par Serge MORAND ${ }^{1}$

(Manuscrit accepté le 25 Avril 2021)

\section{RÉSUMÉ}

Le présent article a pour objet de résumer les connaissances sur les dynamiques d'émergence et d'épidémies de zoonoses liées. Premièrement, en reconnaissant que le processus de domestication est structurant de l'écologie et de l'épidémiologie des zoonoses passées comme des émergences et épidémies actuelles. Deuxièmement, en montrant comment l'augmentation de l'élevage semble être un facteur essentiel dans les dynamiques épidémiologiques et d'émergences de nouvelles zoonoses. Le lien entre élevage, biodiversité et zoonoses s'explique par le fait que les animaux domestiqués sont des ponts épidémiologiques entre la faune sauvage et les humains. Toute augmentation des densités d'animaux de rente et particulièrement dans des pays hébergeant une forte biodiversité est donc supposée accroître les risques d'émergences et d'épidémies de zoonoses. L'absence d'une bonne compréhension de l'émergence et de la transmission des zoonoses ne doit cependant pas nous empêcher de mieux agir afin de prévenir les risques zoonotiques locaux comme les crises sanitaires globales.

Mots-clés : domestication, élevage, faune sauvage, zoonoses, mobilité, émergence de maladies infectieuses, épidémies, One Health

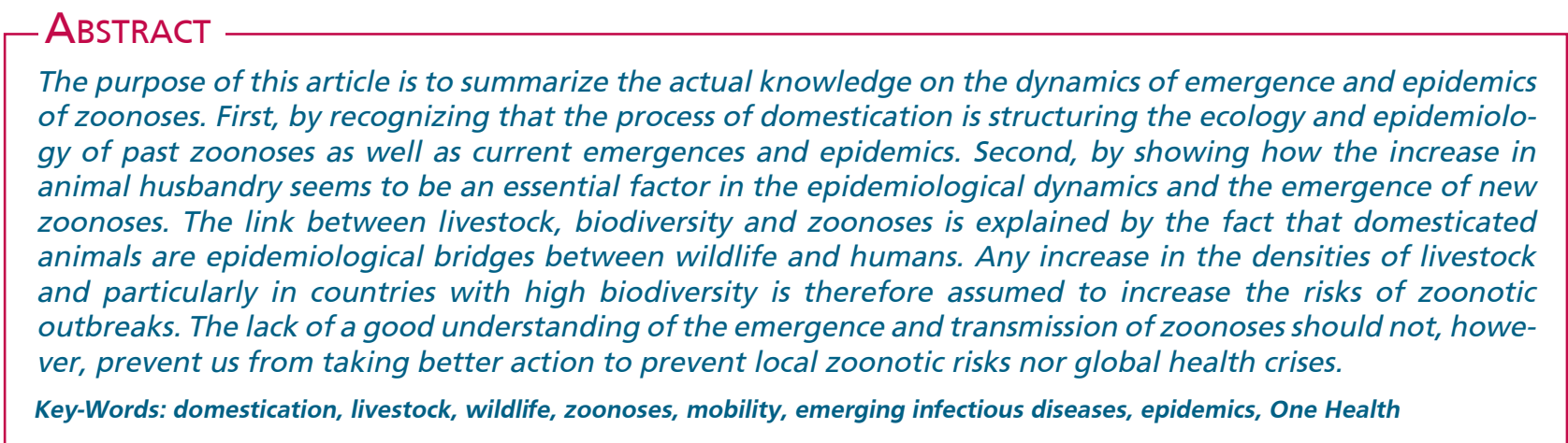

(1) Directeur de Recherche CNRS attaché au CIRAD, Faculty of Veterinary Technology, Kasetsart University, Thaillande, Bangkok, Faculty of Tropical Medicine, Mahidol University, Thaillande, Bangkok. Courriel: serge.morand@umontpellier.fr 


\section{INTRODUCTION}

Les zoonoses représentent plus de $70 \%$ des maladies infectieuses humaines (Cleaveland et al. 2001; Woolhouse \& Gowtage-Sequeria, 2005 ; Vourch et al. 2021). Les agents à l'origine de ces maladies ont été acquis tout au long de l'évolution de l'espèce humaine en et hors du berceau africain, puis au cours de l'histoire récente de l'humanité de la révolution néolithique à l'époque contemporaine. Les travaux en histoire environnementale, en anthropologie, en évolution et écologie nous aident à dresser un tableau de l'histoire globale des zoonoses (McNeill, 1976 ; Diamond, 1997 ; Wolfe et al. 2007 ; Morand, 2016). Les humains ont acquis leurs agents microbiens infectieux et parasitaires par descendance, c'est-à-dire par héritage d'un ancêtre commun avec les autres primates, ou en les acquérant par capture ou débordement («spillover ») d'espèces animales sauvages ou domestiques vivant en proximité écologique. Trois sources majeures d'acquisition des maladies infectieuses et parasitaires ont été suggérées (Morand, 2012) : la source " africaine » où les agents ont été acquis dans le centre de diversification des hominidés et qui, pour nombre d'entre eux, ont accompagné la dispersion et l'expansion des humains modernes hors du continent africain ; la source liée à la «domestication animale » où les agents ont été acquis à partir de la révolution néolithique probablement dans les centres de domestication, ou bien avant cette période en ce qui concerne le chien ; la source liée à la « mondialisation ", reflétant la redistribution géographique des agents pathogènes d'abord le long des premières routes commerciales avec la montée des civilisations agraires, puis par la colonisation européenne de la planète, pour aboutir à la récente industrialisation de la production agricole et la mondialisation accélérée des échanges commerciaux.

Les données archéologiques suggèrent la survenue d'une domestication à grande échelle des animaux depuis environ 12000 ans. La domestication animale avec celle des plantes ont considérablement transformé la biosphère terrestre, modifiant le chemin de l'histoire humaine en permettant l'essor des civilisations agraires (Diamond, 2002). La domestication des animaux a également fortement affecté la santé humaine en favorisant l'émergence puis la propagation des maladies zoonotiques (Pearce-Duvet, 2006). L'importance du bétail et des animaux de compagnie, comme le chien ou le chat, dans partage des maladies infectieuses avec les humains a été soulignée dans de nombreuses études (Cleaveland et al. 2001 ; Lloyd-Smith et al. 2009). Mais c'est avec l'historien de l'environnement William McNeill (1976) que l'hypothèse du rôle essentiel joué par la domestication des animaux dans l'augmentation de la diversité des maladies infectieuses des humains a été clairement formulée. L'historien a été le premier à suggérer que le partage d'agents pathogènes entre les animaux domestiques et les humains est dépendant du temps de vie en commun avec chacun de ces animaux (Morand et al. 2014).

Le nombre de maladies infectieuses émergentes a considérablement augmenté au cours des dernières décennies (Wilcox \& Gubler, 2005 ; Wolfe et al. 2007 ; Jones et al. 2008) comme le nombre d'épidémies de maladies infectieuses zoonotiques ou vectorielles (Smith et al. 2014 ; Morand, 2020). Les causes de ces augmentations en sont attribuées aux changements environnementaux planétaires et à la perte de biodiversité (Keesing et al.
2010 ; Morand \& Lajaunie, 2017 ; Rohr et al. 2019). L'émergence du SARS-CoV-2 en décembre 2019 et la pandémie de COVID-19 résultante, ainsi que la dernière pandémie de A(H1N1) pdm en 2009, interrogent sur l'origine de ces émergences de zoonoses comme de leurs capacités pandémiques.

Le présent article a pour objet de résumer les connaissances sur les dynamiques d'émergence et d'épidémies de zoonoses en explorant deux questions :

- en quoi le processus de domestication est-il structurant de l'écologie et de l'épidémiologie des zoonoses passées comme des émergences et épidémies actuelles?

- en quoi l'augmentation de l'élevage en lien avec son industrialisation récente est-il un facteur important d'explication des dynamiques épidémiologiques et d'émergences de nouvelles zoonoses?

\section{DOMESTICATION ET ZOONOSES}

\section{Les zoonoses et le développement de l'agriculture}

Les données paléontologiques suggèrent que les populations humaines du paléolithique étaient peu affectées par des épidémies mais plus soumises aux maladies parasitaires dues à des vecteurs arthropodes piqueurs ou associées à la consommation de faune sauvage (Morand 2016). L'impact environnemental du développement de l'agriculture a permis aux maladies infectieuses et zoonotiques d'émerger. Le paludisme, la fièvre jaune, les trypanosomiases ou les filarioses ont bénéficié de la déforestation et de l'extension des surfaces agricoles et de l'augmentation des densités humaines (Cohen \& Armelagos 1984). Dans le même temps, la sédentarisation et les densités humaines favorisent la transmission de ces maladies. Ainsi, il faut environ trois kilomètres carrés pour assurer la subsistance d'un chasseur-cueilleur (soit 0,3 humain par $\mathrm{km}^{2}$ ). Avec l'émergence des sociétés agraires, les densités humaines ont été multipliées par dix par rapport à celles des sociétés de chasseurs-cueilleurs, et parfois beaucoup plus lorsque les populations se localisent dans des environnements extrêmement productifs comme les plaines alluviales et les deltas (Cohen \& Armelagos 1984).

\section{Tempo de la domestication et du partage d'agents infectieux}

Morand et al. (2014) ont testé statistiquement l'hypothèse McNeill (1976) sur le rôle historique de la domestication animale dans l'augmentation de la diversité des agents pathogènes humains. La relation positive significative trouvée sur un petit nombre de mammifères domestiqués confirme que le nombre de maladies infectieuses partagées entre ces mammifères et l'espèce humaine, c.à.d. le nombre de zoonoses, est dépendant du temps passé en proximité des humains, c.à.d. du nombre d'années depuis le début de la domestication de chaque espèce.

L'association entre temps de domestication et partage de zoonoses peut se visualiser avec un plus grand nombre d'espèces 
animales et d'agents infectieux et parasitaires en utilisant : (i) les données concernant 888 agents pathogènes (bactéries, virus, protistes, champignons et helminthes) de 23 espèces de mammifères et oiseaux domestiques et de 9 espèces animales de compagnie, apprivoisées et commensales provenant de la base de données de Wardeh et al. (2015); et (ii) les dates estimées de domestication ou de vie en proximité avec les humains, pour les espèces apprivoisées et commensales, à partir des données et des références compilées par Driscoll et al. (2009) et Larson et al. (2014). La Figure 1 montre l'existence d'associations positives entre le nombre d'agents infectieux ou parasitaires partagés avec les humains et le temps de vie en commun pour trois groupes d'espèces : les espèces de mammifères domestiqués, les espèces d'oiseaux domestiqués, et les espèces de mammifères ou d'oiseaux apprivoisées ou commensaux.

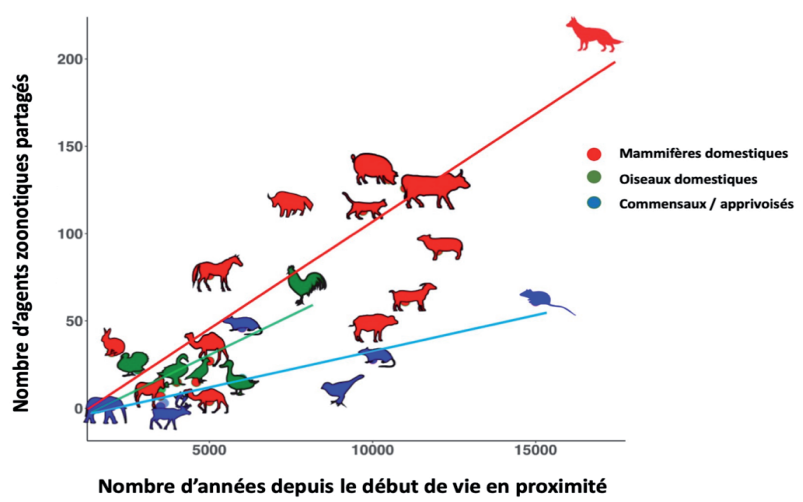

FIgUre 1 : Relations entre temps de domestication ou de vie en commun et partage d'agents zoonotiques. Cette figure a été réalisée grâce (i) aux données concernant 888 agents pathogènes (bactéries, virus, protistes, champignons et helminthes) provenant de la base de données de Wardeh et al. (2015) et pour 23 espèces de mammifères et oiseaux domestiques et 9 espèces animales de compagnie, apprivoisées et commensales; et (ii) aux dates estimées de domestication ou de vie en proximité avec les humains, pour les espèces apprivoisées et commensales, à partir des données et des références compilées par Driscoll et al. (2009) et Larson et al. (2014).

L'hypothèse de McNeill (1976) est encore confirmée mais cette fois avec une communauté d'animaux et de leurs pathogènes partagés beaucoup plus importante. Cette communauté d'animaux, d'humains et d'infections s'est construite sur de longues périodes de vie en commun. Cette observation et les résultats de Morand et al. (2014) soulignent l'importance de la domestication (Pearce-Duvet, 2006) mais également du temps long (Wolfe et al. 2007) dans le développement des zoonoses et des maladies infectieuses n'affectant que les humains mais dont les agents sont originellement issus des animaux. Ces résultats suggèrent également que les animaux récemment domestiqués sont potentiellement des réservoirs d'agents pathogènes non encore émergés ni identifiés. Enfin, ils suggèrent que l'incorporation de nouvelles espèces animales en élevage ou dans le processus de domestication sont porteurs de nouvelles probabilités pour l'émergence de nouveaux agents zoonotiques ou le partage d'agents déjà établis. On pense ainsi à l'infection des visons élevés pour leur fourrure par le SARS-CoV2.

\section{Origine et extension des zoonoses}

La plupart des animaux domestiqués sont originaires d'Asie (Larson et al. 2014 ; Morand, 2016) : Moyen-Orient, Asie de l'Est, Asie Centrale et Asie du Sud et du Sud-Ouest. Peu d'animaux ont été domestiqués en Afrique (pintade, oie et probablement âne), en Europe occidentale (lapin) et dans les Amériques (dindon, cochon d'Inde, lama).

Lors du processus de domestication, les espèces animales ont pu être infectées par les humains. Ainsi, la bactérie Mycobacterium bovis des bovins est issue d'une souche de M. tuberculosis humaine (Smith et al. 2009). L'étude de la structure génétique de Staphylococcus aureus par Weinert et al. (2012) montre un passage de la bactérie des humains aux bovins qui a du se produire il y a 5500 ans au moment de l'expansion des bovins domestiqués. Les résultats de cette étude suggèrent un passage plus récent de S. aureus vers la volaille av. J.-C., bien après leur domestication mais bien avant l'agriculture commerciale.

Cependant, pour la plus grande majorité des agents parasitaires et microbiens, les animaux domestiqués, apprivoisés et commensaux sont les sources d'infection pour les humains. Un travail récent de phylogénie moléculaire du virus de la rougeole conduit par Düx et al. (2020) a permis de revisiter l'histoire ce virus grâce à un poumon, conservé dans le formol, d'une petite fille de deux ans décédée de la rougeole en 1912. La date de divergence du virus de la rougeole et du virus de la peste bovine a pu être ainsi réévaluée. Le virus de la rougeole aurait émergé il y a deux mille quatre cents ans durant la période de l'Antiquité classique, et bien avant la date $11^{\text {ième }}$ siècle précédemment estimée (Furuse et al. 2010).

La dispersion des animaux domestiques hors de leurs centres de domestication s'est accélérée avec les premières routes commerciales (Boivin et al. 2017). Cette dispersion des animaux domestiques a permis l'expansion des maladies zoonotiques, mais aussi des maladies infectieuses spécifiques du bétail comme le virus de la peste bovine, ou encore des maladies infectieuses humains d'origine animale comme la rougeole. Cependant, les études sur la phylogénie et la phylogéographie des agents pathogènes zoonotiques ou non des animaux domestiques sont encore trop peu nombreuses pour nous donner une vision globale de l'origine et de la diffusion des maladies infectieuses.

\section{Le lien à la faune sauvage}

Les animaux domestiques sont des ponts épidémiologiques entre la faune sauvage, en premier lieu, leurs parentés phylogénétiques, et les humains. Une hypothèse est donc que les animaux domestiques favorisent le débordement d'agents infectieux hébergés dans la faune sauvage et en assurent leur amplification.

Cette hypothèse a été testée récemment par Wells et al. (2020) en modélisant statistiquement les modes de partage de 1785 virus $\mathrm{ADN}$ et $\mathrm{ARN}$ entre 725 espèces de mammifères sauvages et domestiques. L'analyse montre que les mammifères domestiques, avec les primates non-humains, occupent les positions les plus centrales dans les réseaux de partage des virus entre mammifères. Les bovins, les porcs domestiques, les chevaux et les moutons occupent une position centrale dans le partage des virus à $\mathrm{ADN}$. Ces résultats confirment que les animaux de 
rente, économiquement importants (Thornton, 2010) contribuent fortement au partage de virus entre la faune et les humains (voir également Johnson et al. 2020). A noter que les chauves-souris occupent une position centrale dans le partage des virus à ARN, mais que nombre de ces virus sont aussi associés à des animaux domestiques comme le virus Nipah avec le porc domestique, le virus Hendra avec le cheval, le MERS-CoV avec le dromadaire et le SAR-Cov1 avec la civette. Ces résultats sont à mettre en relation avec l'analyse de Dunn et al. (2010) sur le fardeau des maladies infectieuses humaines par pays. Cette étude montre que la diversité des agents infectieux et parasitaires humains est positivement associée à la richesse en espèces d'oiseaux et de mammifères par pays, confirmant que la diversité des agents pathogènes humains est plus élevée dans les régions intertropicales que dans les régions tempérées (Guernier et al. 2004). Vourch et al. (2021) montrent l'existence d'une corrélation similaire entre la diversité des maladies zoonotiques affectant les populations humaines et la diversité des espèces animales par pays. Un pays riche en biodiversité est également un pays riche en agents zoonotiques.

Les résultats des études succinctement présentées ci-dessus suggèrent une nouvelle hypothèse : du fait que les animaux domestiqués sont des ponts épidémiologiques entre la faune sauvage et les humains, toute augmentation des densités d'animaux de rente dans des pays hébergeant une forte biodiversité devrait accroître les risques zoonotiques (émergences et épidémies de zoonoses).

\section{LA GRANDE ACCÉLÉRATION DES ÉPIDÉ- MIES DE ZOONOSES}

\section{Un problème de mobilité}

Le nombre d'épidémies de maladies zoonotiques et à transmission vectorielle a augmenté au cours des dernières décennies selon la base de données GIDEON. Il y a de plus en plus d'épidémies de maladies infectieuses qu'elles soient zoonotiques ou vectorielles (Morand \& Walther, 2020 ; Morand \& Lajaunie, 2021) avec des épidémies qui tendent à se globaliser de plus en plus à partir du début des années 1960 (Morand \& Walther, 2020). Il faut aussi noter que certaines émergences à potentiel pandémique n'ont pas eu de suite et leurs agents, "humanisés", semblent avoir disparu comme dans le cas du SARS-Cov1.

L'augmentation des transports de voyageurs et de marchandises semble être l'explication de cette augmentation des épidémies et pandémies. Selon les données de la Banque Mondiale, les transports de voyageurs et de marchandises ont augmenté de manière exponentielle sur les dernières décennies. Le nombre total de passagers aériens est passé d'environ 332 millions en 1970 à plus de 4 milliards en 2017 (une augmentation de $1250 \%$ ), tandis que le volume total de fret aérien est passé d'environ 15500 millions de tonnes par km parcouru (MT-km) en 1970 à plus de 220000 MT-km en 2017 (une augmentation de $1400 \%)$. Les arrivées de touristes internationaux dans le monde ont bondi de 25 millions d'arrivées en 1950 à 1400 millions en 2016 (une augmentation de 5600 \%) et les projections de l'Organisation Mondiale du Tourisme (OMT) donnent un chiffre de 1,8 milliard de touristes d'ici 2030. De la même manière, le produit intérieur brut mondial par habitant est passé de 805 dollars américains en 1970 à 10778 dollars américains en 2017 (une augmentation de 1300\%). La corrélation observée entre le nombre croissant d'épidémies de maladies infectieuses et l'augmentation des transports, comme de la richesse économique, est en faveur de l'hypothèse selon laquelle la mondialisation économique accélère la mondialisation des maladies infectieuses (Morand \& Walther 2020).

Les modélisations mathématiques montrent qu'un accroissement de la mobilité se traduit par une propagation plus rapide et plus globale d'une épidémie (Hufnagel et al. 2004) et que seules les mesures qui diminuent la mobilité ralentissent effectivement la propagation des agents infectieux (Hollingsworth et al. 2006). Les modèles suggèrent également que les réductions précoces des déplacements mises en ouvre au début des épidémies réduisent considérablement le nombre de villes dans le monde touchées par une épidémie majeure (Cooper et al. 2006).

Cependant, une réduction du commerce mondial et des voyages n'est pas suffisante pour empêcher l'apparition et la transmission locale d'agents infectieux. Les conditions locales prévalent dans l'apparition de nouvelles maladies infectieuses émergentes et, parmi ces conditions, l'agriculture présente un intérêt particulier dans le contexte d'épidémies récurrentes, à l'exemple du A(H1N1) pdm ou du H5N1.

La démographie humaine trouve également toute sa place en tant que facteur favorisant la propagation et l'amplification des maladies infectieuses. Cependant, pas de manière simple en ne prenant en compte que la taille de population ou son taux de croissance, mais en soulignant l'importance d'une transition majeure dans l'histoire de l'humanité. Plus de la moitié de la population mondiale (54\% en 2014 ) vit maintenant dans les villes et les projections donnent une population urbaine totale de plus de 5 milliards d'urbains à l'horizon 2030. La transition vers une civilisation urbaine s'accompagne de nombreux changements, et d'abord celui d'un changement d'alimentation plus riche en protéines animales particulièrement important dans les villes des pays récemment industrialisés. Les centres urbains anciens et nouveaux sont connectés entre eux par un réseau en pleine expansion d'aéroports et de routes permettant la circulation des personnes, des biens, et des agents infectieux.

\section{Un problème d'accroissement de l'élevage}

L'augmentation spectaculaire du cheptel au cours des dernières décennies apparaît comme une cause majeure du déclin de la biodiversité et de l'altération des habitats naturels (Steinfeld et al. 2006 ; Reid et al. 2010 ; Alkemade et al. 2013). Selon les données de la FAO (Organisation des Nations Unies pour l'Agriculture et l'Alimentation), le nombre de bovins a dépassé le milliard et 600 millions en 2019 alors qu'il était inférieur au milliard en 1960. Dans le même temps, le nombre de porcs est passé de 500 millions à 1,5 milliard et celui des poulets de 5 milliards à 30 milliards (sans compter les poules pondeuses). De nos jours, le bétail a une biomasse totale plus élevée que la biomasse de tous les humains (Smil 2002, Bar-On et al. 2018), tandis que le nombre de volailles (poulets, poules, canards, oies) pourrait être d'une magnitude identique au nombre d'oiseaux sauvages. Le nombre total d'oiseaux avait été estimé entre 200 et 300 milliards d'individus en 1997 (Gaston \& Blackburn, 1997), 
bien avant l'accélération du déclin de leur abondance (WWF 2020).

L'augmentation du nombre d'épidémies de maladies infectieuses affectant les humains, comme d'ailleurs l'augmentation du nombre d'épidémies affectant les animaux d'élevage, du bétail, est à mettre en corrélation avec l'augmentation du cheptel bovin (Morand 2020). L'accroissement temporel du nombre d'animaux de rente apparaît contribuer à l'accroissement du nombre d'épidémies, comme à l'accroissement du nombre d'espèces sauvages menacées (Morand 2020).

L'accroissement de l'élevage s'explique par la croissance de la population humaine, par les changements de régimes alimentaires liés à la croissance économique et à l'occidentalisation des habitudes, par l'industrialisation agricole et son impact sur les habitats naturels ou gérés traditionnellement, par l'intégration dans le commerce mondial, mais aussi par les valeurs culturelles de l'élevage, qui peuvent varier d'un pays à l'autre.

L'expansion mondiale de l'élevage en menaçant la biodiversité, en modifiant l'utilisation des terres et en homogénéisant les paysages, accroît sérieusement les ponts épidémiologiques entre la faune sauvage et les humains.

\section{DISCUSSION}

L'émergence est un problème local de transmission d'un agent infectieux. Pour le cas des zoonoses, l'émergence se produit à l'interface entre animaux sauvages et / ou animaux domestiques et humains dans des environnements en changements rapides sous les effets combinés du changement climatique, de la modification des habitats, de l'intensification agricole et des pertes de la biodiversité. Nous sommes cependant encore bien loin d'avoir une compréhension complète des processus écologiques qui sous-tendent la dynamique des maladies infectieuses émergentes. Alors que l'accent est souvent mis sur la faune sauvage dans l'étude de l'émergence (Keesing et al. 2010), relativement peu d'études ont pris en compte la complexité du phénomène d'émergence qui doit considérer les capacités évolutives des agents infectieux (Bonneaud \& Longdon 2020, Easterday 2020), le compartiment des animaux domestiques et sauvages (Morand 2020) ou celui des changements d'habitat (Morand \& Lajaunie 2021). Un effort de recherche accru doit examiner les zoonoses dans la dynamique des changements des interfaces environnement, biodiversité, élevage et sociétés.

De cet état des connaissances présentées rapidement, il convient de retenir deux enseignements de compréhension et d'étude du fait zoonotique :

- la nécessité d'intégrer le temps long, celui qui a contraint l'écologie évolutive des zoonoses, dans le temps court de la transmission épidémiologique, de l'épidémie et de la pandémie ;

- l'importance d'articuler le global, celui des changements planétaires facteurs de forçage sur le local, et du local territoire des émergences avec des conséquences potentiellement globales.

\section{Biodiversité et zoonoses}

Comme le soulignent Johnson et al. (2015), deux perspectives paradoxales se sont dégagées des études diverses concernant les liens entre biodiversité et transmission de maladies zoonotiques.
La première perspective fait référence à l'hypothèse « la diversité engendre la diversité " et postule que toute augmentation de la diversité des hôtes est positivement corrélée à la diversité globale des parasites (Hechinger \& Lafferty, 2005). Cette perspective prend appui sur les travaux déjà cités de Dunn et al. (2010) confortés par les analyses plus récentes sur les zoonoses (Vourch et al. 2021). Mais cette perspective ajoute qu'une forte diversité accroît les risques de transmission de maladies (Hechinger \& Lafferty, 2005), ce qui est loin d'être démontré. La seconde perspective fait référence à l'hypothèse de dilution ou hypothèse de la relation négative entre biodiversité et transmission. Cette perspective postule que les pertes de biodiversité favorisent la transmission d'agents pathogènes (Keesing et al. 2006).

Les liens potentiels entre zoonoses et biodiversité sont présentés de manière peu compréhensible pour les secteurs de la santé publique et de la santé animale (Morand 2020 ; Rohr et al. 2020). Un premier écueil réside dans ce qui est mesuré. S'agit-il d'une mesure statique de la présence d'un agent infectieux (ou de la diversité des agents infectieux), de la maladie qu'il provoque (ou qu'ils provoquent), de la prévalence dans un réservoir ou vecteur donné ? Est-ce une mesure dynamique comme une épidémie, qui révèle de la description d'une chaîne de transmission, ou de la présence endémique d'une maladie? Un deuxième écueil est l'échelle écologique comme le souligne Magnusson et al. (2020). S'agit-il d'une étude locale qui a analysé la transmission de la maladie dans un socio-écosystème spécifique? Ou s'agit-il d'une étude qui s'intéresse davantage à la dynamique globale des maladies infectieuses entre les nations? L'absence d'une bonne compréhension de l'émergence et de la transmission des zoonoses ne doit pas nous empêcher de mieux agir afin de prévenir les risques sanitaires locaux comme les crises sanitaires globales.

\section{Réinsérer l'élevage dans les territoires}

Les crises sanitaires à répétition ont des conséquences économiques et sociales importantes, notamment par leurs effets sur les systèmes de santé et sur les systèmes de production du fait l'intégration des pays dans une économie mondialisée. Les réponses à l'emballement des crises sanitaires n'ont été jusqu'à présent que la surveillance et la biosécurité. Sans pour autant nier leur importance, il faut reconnaître qu'elles n'ont pas démontré leur capacité à prévenir les nouvelles crises. Les gestions des crises sanitaires affectant les élevages contribuent à favoriser l'industrialisation des élevages et de l'agriculture du fait des coûts de biosécurité sans que les coûts environnementaux, sociaux et sanitaires de l'agro-industrialisation ne soient réellement pris en compte.

Le temps est certainement venu de repenser l'élevage dans son insertion au local et dans ses contributions au développement territorial. L'agroécologie, l'agroforesterie sont des approches permettant d'accroitre la résilience des territoires de productions agricoles ou forestières face aux risques environnementaux, dont le dérèglement climatique, tout en assurant la sécurité alimentaire et sanitaire. Une même démarche concernant l'élevage doit être encouragée. Ce sont de nouvelles formes d'échanges entre les territoires et leurs environnements urbains proches qu'il s'agit de promouvoir par la mise en place d'une agroéconomie circulaire. Cette agroéconomie circulaire est 
seule à même d'enclencher la transition agroécologique souhaitable permettant une utilisation durable de ressources comme la production primaire ou l'eau.

\section{Repenser la gouvernance de la santé et de l'environnement pour mieux répondre aux pandémies}

Une pandémie majeure était prévisible - et prévue. L’Organisation Mondiale de la Santé l'a d'ailleurs dénommée la " maladie X » et a listé les agents infectieux potentiels qui pouvaient en être à l'origine, mais sans trop explorer les conditions socio-écologiques de leur potentiel d'émergence.

Les épidémies et émergences de zoonoses sont les manifestations d'un dysfonctionnement de nos liens aux animaux, sauvages et domestiques. L'approche "One Health » n'a encore que peu pris en compte la santé des écosystèmes (Destoumieux-Garzón et al. 2018) portée par le Programme des Nations Unies pour l'Environnement (de Garine-Wichatitsky et al. 2020). L'approche "Santé dans toutes les politiques " préconisée par l'OMS est encore peu mise en œuvre, ce qui explique le peu de dialogue et d'actions intersectoriels lors des crises sanitaires. Tous les écueils évoqués ci-dessus imposent de mieux traduire les données scientifiques des domaines sectoriels de la conserva- tion, de l'écologie de la transmission, de la santé publique et de la santé animale à un moment où l'intégration des approches est préconisée, avec un renouvellement de l'approche "One Health " intégrant l'environnement avec l'élargissement de la tripartite FAO-OIE-OMS à la quadripartite avec le PNUE, l'approche "Ecohealth" ou bien "Planetary Health" (Whitmee et al. 2015 ; Morand et al. 2020).

\section{CONCLUSION}

La prochaine émergence est certaine et pourtant nous ne pouvons pas prédire quel agent, quel réservoir animal, quel lieu géographique, ni quand elle aura lieu. Les systèmes de santé publique et de santé vétérinaire sont donc contraints d'accroître leurs capacités de surveillance comme leurs capacités de résilience en cas d'épidémies ou de pandémies. L'urgence est de s'attaquer aux causes favorisant les émergences des zoonoses et leurs transmissions à large échelle. Pour cela, nous devons admettre la complexité des facteurs en jeu, nécessitant des recherches pluridisciplinaires, et la complexité des actions publiques nécessitant une intersectorialité renouvelée. Cette urgence nécessite également de renforcer le dialogue entre science et décisions politiques.

\section{REMERCIEMENTS}

Ce travail a bénéficié $d u$ soutien $d u$ projet ANR FutureHealthSEA "Predictive scenarios of health in Southeast Asia" (ANR-17-CE35-0003-01) et du projet "Animal Innovative Health" par l'Agence de Coopération Internationale de Thaïlande (Thailand International Cooperation Agency).

\section{BIBLIOGRAPHIE}

- Alkemade R, Reid RS, van den Berg M, de Leeuw J, Jeuken M. Assessing the impacts of livestock production on biodiversity in rangeland ecosystems. Proc Nat Acad Sci USA 2013; 110 : 20900-20905.

- Bar-On YM, Phillips R, Milo R. The biomass distribution on Earth. Proc Nat Acad Sci 2018; 115: 6506-6511.

- Boivin N, Crassard R, Petraglia MD. Human dispersal and species movement: from prehistory to the present. Cambridge, United Kingdom: Cambridge University Press, 2017.

- Bonneaud C, Longdon B. Emerging pathogen evolution: Using evolutionary theory to understand the fate of novel infectious pathogens. EMBO Rep. 2020; 21(9):e51374.

- Cleaveland S, Laurenson MK, Taylor LH. Diseases of humans and their domestic mammals: Pathogen characteristics, host range and the risk of emergence. Phil Trans R Soc B. 2001; 356: 991-999.
- Cohen M.N. \& Armelagos G.J. Paleopathology at the origin of agriculture. Orlando: Academic Press, 1984.

- Cooper BS, Pitman RJ, Edmunds WJ, Gay NJ. Delaying the international spread of pandemic influenza. PLoS Medicine 2006; 3: e212.

- de Garine-Wichatitsky M, Binot A, Ward JR, Caron A, Perroton A, Ross $\mathrm{H}$ et al. "Health in" and "Health of" Social-Ecological Systems: a practical framework for the management of healthy and resilient agricultural and natural ecosystems. Front Public Health 2021; 8: 982.

- Destoumieux-Garzón D, Mavingui P, Boetch G, Boissier J, Darriet F, Duboz $\mathrm{P}$ et al. The One Health concept: 10 years old and a long road ahead. Front Vet Sci 2018; 5: 10.3389/fvets.2018.00014

- Diamond J. Guns, germs and steel: The fates of human Societies. New York: Norton, 1997.

- Diamond J. Evolution, consequences and future of plant and animal domestication. Nature 2002; 418: 700-707.
- Driscoll C, Macdonald D.W, O’Brien SJ. From wild animals to domestic pets, an evolutionary view of domestication. Proc Natl Acad Sci USA 2009; 106: 9971-9978

- Dunn RR, Davies TJ, Harris NC, Gavin MC. Global drivers of human pathogen richness and prevalence. Proc R Soc B 2010; 277: 2587-2595.

- Düx A, Lequime S, Patrono LV, Vrancken B, Boral S, Gogarten JF et al. Measles virus and rinderpest virus divergence dated to the sixth century BCE. Science 2020; 368: 1367-1370

- Easterday WR. The first step in the success or failure of emerging pathogens. Proc Natl Acad Sci USA 2020; 117: 29271-29273

- Furuse Y, Suzuki A, Oshitani H. Origin of measles virus: divergence from rinderpest virus between the 11th and 12th centuries. Virol J 2010; 7: 52. Gaston KJ \& Blackburn TM. How many birds are there? Biodiv Cons 1997; 6: 615-625. 
- GIDEON, Global Infectious Disease and Epidemiology Network. Disponible à : www.gideononline.com (consulté le 18/04/2021).

- Guernier V, Hochberg ME, Guégan JF. Ecology drives the worldwide distribution of human diseases. PLoS Biology 2004; 2: 740-746.

- Hechinger RF \& Lafferty KD. Host diversity begets parasite diversity: bird final hosts and trematodes in snail intermediate hosts. Proc R Soc Lond B 2005; 272: 1059-1066.

- Hollingsworth TD, Ferguson NM, Anderson RM. Will travel restrictions control the International spread of pandemic influenza? Nature Medicine 2006; 12: 497-499.

- Hufnagel L, Brockmann D, Geisel T. Forecast and control of epidemics in a globalized world. Proc Nat Acad Sci USA 2004; 101: 15124-15129.

- Johnson CK, Hitchens PL, Pandit PS, Rushmore J, Evans TS, Young CCW et al. Global shifts in mammalian population trends reveal key predictors of virus spillover risk. Proc R Soc Lond B 2020; 287: 20192736.

- Jones KE, Patel NG, Levy MA, Storeygard A, Balk D, Gittleman JL et al. Global trends in emerging infectious diseases. Nature 2008; 451: 990-993.

- Keesing F, Belden LK, Daszak P, Dobson A, Harvell CD, Holt RD et al. Impacts of biodiversity on the emergence and transmission of infectious diseases. Nature 2010; 468: 647-652.

- Keesing F, Holt RD, Ostfeld RS. Effects of species diversity on disease risk. Eco Lett 2006; 9: 485-498.

- Larson G., Piperno DR, Allaby RG, Purugganan MD, Andersson L, Arroyo-Kalin $\mathrm{M}$ et al. Current perspectives and the future of domestication studies. Proc Natl Acad Sci USA 2014; 111: 6139-6146.

- Lloyd-Smith JO, George D, Pepin KM, Pitzer VE, Pulliam JRC, Dobson AP et al. Epidemic dynamics at the human-animal interface. Science 2009; 326 : 1362-1367.

- Magnusson M, Fischhoff IR, Ecke F, Hornfeldt B, Ostfeld RS. Effect of spatial scale and latitude on diversity-disease relationships. Ecology 2020; 101: e02955. McNeill WH. Plagues and people. New York: Anchor Press, 1976.
- Morand S. La Prochaine peste. Une histoire globale des sociétés et de leurs épidémies. Paris : Éditions Fayard, 2016.

- Morand S. Emerging diseases, livestock expansion and biodiversity loss are positively related at global scale. Bio Cons 2020; 248: 108707.

- Morand S, Guégan, J-F, Laurans Y. From One Health to Ecohealth, mapping the incomplete integration of human, animal and environmental health. Iddri, Issue Brief 2020; N04/20.

- Morand S \& Lajaunie C. Biodiversity and Health. Linking Life, Ecosystems and Societies. London: Elsevier, 2017.

- Morand S \& Lajaunie C. Outbreaks of vector-borne and zoonotic diseases are associated with changes in forest cover and oil palm expansion at global scale. Front Vet Parasitol 2021; 24: 661063.

- Morand S, McIntyre, KM, Baylis M. Domesticated animals and human infectious diseases of zoonotic origins: domestication time matters. Inf Gen Evol 2014; 24: 76-87.

- Morand S \& Walther B. The accelerated infectious disease risk in the Anthropocene: more outbreaks and wider global spread. BioRxiv 2020; https://doi.org $\angle 10.1101 / 2020.04 .20 .049866$

- Pearce-Duvet JMC. The origin of human pathogens: evaluating the role of agriculture and domestic animals in the evolution of human disease. Biol Rev 2006; 81: 369-82.

- Reid RS, Bedelian C, Said MY, Kruska RL, Mauricio RM, Castel V et al. Global livestock impacts on biodiversity. In: Livestock in a changing landscape. drivers, consequences, and responses, vol 1. Steinfeld H, Mooney HA, Schneider F, Neville LE, editors. Washington DC: Island Press; 2010, pp 111-138.

- Rohr JR, Barrett CB, Civitello DJ, Craft ME, Delius B, DeLeo GA et al. Emerging human infectious diseases and the links to global food production. Nature Sustainability 2019; 2 : 445-456.

- Rohr JR, Civitello DJ, Halliday FW, Hudson PJ, Lafferty KD, Wood CL et al. Towards common ground in the biodiversity-disease debate. Nature Ecol 2010; 4: 24-33.

- Smil V. The Earth's biosphere. evolution, dynamics, and change. Cambridge MA: Massachusetts Institute of Technology, 2002.
- Smith KF, Goldberg M, Rosenthal S, Carlson L, Chen J, Chen C et al. Global rise in human infectious disease outbreaks. J R Soc Interface 2014; 11: 20140950.

- Steinfeld H, Gerber P, Wassenaar TD, Castel C, Rosales M, de Haan C. Livestock's Long Shadow: Environmental Issues and Options. Roma: Food and Agriculture Organization of the United Nations, 2006.

- Thornton PK. Livestock production: recent trends, future prospects. Philos Trans R Soc Lond B Biol Sci 2010; 365 : 2853-2867.

- Vourch G, Morand S., Moutou F, Jourdain E. Les zoonoses - Enjeux Sciences. Versailles : Quae, 2021.

- Wardeh M, Risley C, McIntyre MK, Setzkorn C, Baylis M. Database of host-pathogen and related species interactions, and their global distribution. Sci Data 2015; 2: 150049.

- Wells K., Morand S., Wardeh M., Baylis M. Distinct spread of DNA and RNA viruses among mammals amid prominent role of domestic species. Global Ecol Biogeo 2020; 29: 470-481.

- Weinert LA, Welch JL, Suchard MA, Lemey P, Rambaut A, Fitzgerald J.R. Molecular dating of human-to-bovid host jumps by Staphylococcus aureus reveals an association with the spread of domestication. Biol Lett 2012; 8: 829-832.

- Wilcox BA \& Gubler DJ. Disease ecology and the global emergence of zoonotic pathogens. Environ Health Prev Med 2005; 10:263-272.

-Whitmee S, Haines A, Beyrer C, Boltz F, Capon AG, de Souza Dias BF et al. Safeguarding human health in the Anthropocene epoch: report of The Rockefeller Foundation-Lancet Commission on planetary health. Lancet 2015; 386:1973-2028.

- Wolfe ND, Dunavan CP, Diamond J. Origins of major human infectious diseases. Nature 2007; 447: 279-283.

-Woolhouse MEJ \& Gowtage-Sequeria S. Host range and emerging and reemerging pathogens. Emerg Infect Dis 2005; 11: 1842-1847.

-WWF. Living Planet Report Bending the curve of biodiversity loss. Editors Almond REA, Grooten M, Petersen T. Gland, Switzerland: WWF, 2020. 